\title{
Current vortices in aromatic carbon molecules
}

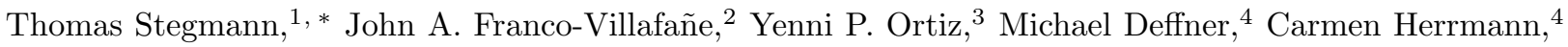 \\ Ulrich Kuhl, ${ }^{5}$ Fabrice Mortessagne, ${ }^{5}$ Francois Leyvraz, ${ }^{1,6}$ and Thomas H. Seligman ${ }^{1,6}$ \\ ${ }^{1}$ Instituto de Ciencias Físicas, Universidad Nacional Autónoma de México, 62210 Cuernavaca, Mexico \\ ${ }^{2}$ CONACYT - Instituto de Física, Universidad Autónoma de San Luis Potosí, 78290 San Luis Potosí, Mexico \\ ${ }^{3}$ Instituto de Física, Universidad Nacional Autónoma de México, 04510 Mexico City, Mexico \\ ${ }^{4}$ Institute for Inorganic and Applied Chemistry, University of Hamburg, 20146 Hamburg, Germany \\ ${ }^{5}$ Université Côte d'Azur, CNRS, Institut de Physique de Nice, 06100 Nice, France \\ ${ }^{6}$ Centro Internacional de Ciencias, 62210 Cuernavaca, Mexico
}

(Dated: January 23, 2020)

\begin{abstract}
The local current flow through three small aromatic carbon molecules, namely benzene, naphthalene and anthracene, is studied. Applying density functional theory and the non-equilibrium Green's function method for transport, we demonstrate that pronounced current vortices exist at certain electron energies for these molecules. The intensity of these circular currents, which appear not only at the anti-resonances of the transmission but also in vicinity of its maxima, can exceed the total current flowing through the molecular junction and generate considerable magnetic fields. The $\pi$ electron system of the molecular junctions is emulated experimentally by a network of macroscopic microwave resonators. The local current flows in these experiments confirm the existence of current vortices as a robust property of ring structures. The circular currents can be understood in terms of a simple nearest-neighbor tight-binding Hückel model. Current vortices are caused by the interplay of the complex eigenstates of the open system which have energies close-by the considered electron energy. Degeneracies, as observed in benzene and anthracene, can thus generate strong circular currents, but also non-degenerate systems like naphthalene exhibit current vortices. Small imperfections and perturbations can couple otherwise uncoupled states and induce circular currents.
\end{abstract}

\section{INTRODUCTION}

In the last decades, the structural size of electronic circuits has been reduced enormously. This is manifested impressively by the fact that the first field-effect transistor, invented by W. Schockley et al. in 1947, had the size of the palm of a hand, while nowadays, billions of transistors of size of a few nanometers are packed on a single chip. This miniaturization of electronic circuits gave rise to the question if even a single molecule can be used as the functional element of an electronic device [1] and founded eventually the active research field of molecular electronics, see for example Refs. 22 4] for an overview. One of the main research topics of molecular electronics is to understand the current flow through a molecular junction. A recent milestone has been the observation of quantum interference in molecular junctions [5] and its theoretical understanding 9 14. However, the local current flow, i.e. the spatial distribution of the current in the molecule, has received less attention, see Refs. 1543 for recent work. This can be attributed to the fact that - to the best of our knowledge - the local current flow in molecular junctions has been measured only indirectly by nuclear magnetic resonance [44, 45] but not yet directly.

One remarkable theoretical prediction with respect to the local current flow is the fact that aromatic molecules can show at certain electron energies pronounced current vortices, which can exceed even the total current flowing through the molecular junction. These predictions have been made by means of state-of-the-art theory, com- bining Kohn-Sham density functional theory (DFT) for the electronic structure of the molecular junction with the non-equilibrium Green's function method (NEGF) for the electron transport [18, 19, 37. It has also been shown that the current vortices persist even if the molecular junction is described by a more elementary theory, namely a tight-binding Hückel Hamiltonian, where electron-electron interactions are not taken into account, and commonly only a single atomic orbital per atom is used [16, 20 22, 41, 42. Also a mixture of both approaches, the density functional based tight-binding (DFTB) method, predicts vortices in the local current flow [29]. Thermoelectric driven ring currents have also been reported recently within a tight-binding model [46]. Therefore, the observed circular currents are not a subtle many-body phenomenon but a more robust general property of ring structures.

In this article, we analyze the current vortices in three small aromatic carbon molecules, namely benzene, naphthalene and anthracene molecules, see Figure 1 In particular, we show that circular currents can not only be observed close to anti-resonances in the transmission function but also in the vicinity of transmission maxima and hence, generate a magnetic field. Indeed, in the following, we shall use a definition of circular current, see [20, 42], which corresponds precisely to the source of this magnetic field. More important, here we give experimental indications of current vortices in ring structures analogous to the molecular structures. The formal equivalence of the Helmholtz equation for electromagnetic waves and the non-interacting Schrödinger equation for quantum 


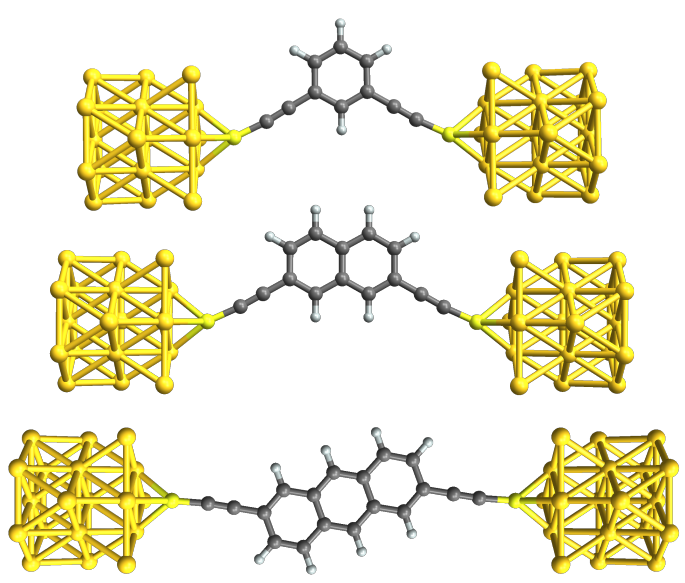

Figure 1. Benzene (top), naphthalene (middle) and anthracene (bottom) molecules are connected via two carbon spacer atoms and a sulfur atom to gold clusters (19 atoms, fcc lattice symmetry) and hence, form a molecular junction. Benzene and naphthalene are shown with leads in the meta position, while for anthracene the leads are in the para position. In our studies, both lead configurations are taken into account for all molecules.

wave functions allows to emulate quantum systems in microwave cavities [47 49. This idea can be used to realize tight-binding systems by networks of dielectric microwave resonators [50, 51, as models for graphene [52[56] and molecules [57. Here we apply this method to emulate the current flow in aromatic carbon molecules by means of a network of dielectric resonators, see Figure 2. By measuring the amplitude and phase of the electromagnetic field on each microwave resonator, we can investigate experimentally the local current flow in these structures. The emulation experiments show clearly circular currents as observed in the quantum calculations.

\section{SYSTEMS \& METHODS}

We study the current flow in aromatic carbon molecules, to be precise, benzene, naphthalene and anthracene. These molecules are attached to gold clusters using two additional carbon spacer atoms and a sulfur atom, see Figure 1. The gold clusters are built up of 19 atoms in fcc lattice symmetry, though the precise configuration of the gold clusters does not change qualitatively the current flow in the molecular junction.

\section{Quantum transport calculations}

In order to calculate the current flow through the molecular junction, the structure is optimized first by means of DFT as implemented in the Gaussian09 software package [58. The exchange-correlation functional

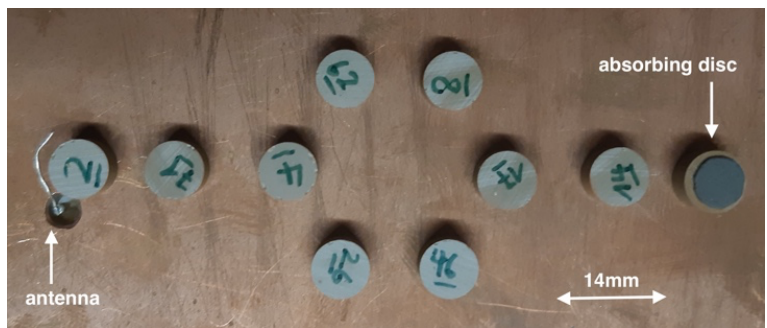

Figure 2. Photo of the microwave emulation experiment for the benzene molecule in the para configuration of the leads. Dielectric cylindrical resonators model the carbon atoms. The source antenna, which injects microwaves, is shown at the left end. A resonator with an absorbing material glued to its top models the drain at the right end. The electromagnetic field (or wave function) is measured on each resonator by a movable antenna through the metallic top plate (both not shown in the photo), which allows to calculate the local transmissions.

B3LYP and the basis set LANL2MB are used to describe the system. On this basis, the current flow is calculated by means of the NEGF method as implemented in the Artaios software package [59]. It calculates the Green's function of the scattering region (i.e. the molecular junction without the gold clusters)

$$
G(E)=\left(E S-H-\Sigma_{S}-\Sigma_{D}\right)^{-1},
$$

using the effective single-particle Kohn-Sham Hamiltonian $H$ and the overlap matrix $S$ from the DFT optimization. The self-energies $\Sigma_{S / D}$ describe the effect of the source and drain reservoirs, which are attached through the gold clusters in order to inject and extract electrons. These reservoirs are modelled within the wideband approximation 60. Using the Green's function, we study the overall transport by means of the transmission function

$$
T(E)=4 \operatorname{Tr}\left(\operatorname{Im}\left(\Sigma_{S}\right) G \operatorname{Im}\left(\Sigma_{D}\right) G^{\dagger}\right),
$$

as well as the local transmissions between the atoms $i$ and $j$

$$
T_{i j}(E)=\operatorname{Im}\left(H_{i j}^{*} G_{i j}^{n}\right),
$$

where the correlation function is given by

$$
G^{n}=G \operatorname{Im}\left(\Sigma_{S}\right) G^{\dagger} .
$$

Note that in equation (3) we have assumed implicitly the sum over pairs of local basis functions which belong to the two atoms. In order to obtain the current from the transmission and the local transmissions, one has to integrate these functions within the bias voltage window. Details of the methods can be found in Refs. [4, 61, 63.

\section{Microwave emulation experiments}

Non-interacting quantum systems can be emulated by means of a network of dielectric microwave resonators 
[50 57. Here, we use this technique to gain insight into the local current flow in aromatic carbon molecules. In the experiment, the carbon atoms are represented by identical cylindrical resonators $(5 \mathrm{~mm}$ height, $4 \mathrm{~mm}$ radius, refractive index $n \approx 6$ ), that are placed between two metal plates, see Figure 2. The nearest neighbor distance of the resonators is on average $14 \mathrm{~mm}$, while their precise distance ratios reflect the interatomic distances found by the DFT optimization of the molecule. The coupling strength between neighboring resonators decays approximately exponentially, as shown previously [54, 57. Such an exponential decay is a realistic model for slightly varying carbon-carbon bond distances, as shown for deformed graphene 64. The hydrogen atoms are not taken into account explicitly, as we assume that the current is carried predominantly by the $\pi$ electron system that is formed by the $2 p_{z}$ orbitals of the carbon atoms. Hence, these orbitals, as well as their interactions, are emulated by a microwave resonator network.

The effects of the gold clusters and sulfur atoms are taken into account indirectly by the antenna at the left end that injects microwaves and a resonator with an absorbing material glued to its top at the right end which serves as the drain. The microwaves are injected as transverse electrical modes around the frequency $\nu_{0}=$ $6.65 \mathrm{GHz}$, where the individual resonators have an isolated resonance. Note that in contrast to the theory, the source (injecting antenna) and drain (absorbing resonator) are modelled differently in the emulation experiment, compare Figure 1 and Figure 2 The other components of the molecular junction (gold, sulfur and hydrogen atoms), their complicated interactions as well as correlations between the electrons are taken into account (approximately) in the DFT-NEGF calculations but not in the emulation experiments. To which extent molecules that are composed of different types of atoms can be modelled by means of different types of microwave resonators will be addressed in our future work.

In order to determine the local transmission in the resonator network, the electromagnetic field (or wave function) $\psi(\boldsymbol{r})$ is measured on each resonator by means of a movable antenna through the metallic top plate. Starting from the definition of the probability current density $\boldsymbol{j}(\boldsymbol{r})=\frac{\hbar}{m} \operatorname{Im}\left(\psi^{*}(\boldsymbol{r}) \nabla \psi(\boldsymbol{r})\right)$ and discretizing it, we obtain for the transmission between two resonators at frequency $\nu$ 65]

$$
T_{i j}^{\mathrm{mw}}(\nu)=\operatorname{Im}\left(d_{i j}^{-1} \psi_{i}^{*} \psi_{j}\right),
$$

where $d_{i j}$ is the distance between the resonators $i$ and $j$. Note that in the case of a non-interacting system, equation (5) is formally equivalent to equation (3), because $G_{i j}^{n} \sim \psi_{i}^{*} \psi_{j}$ and $d_{i j}$ is proportional to the coupling matrix elements $H_{i j}$.
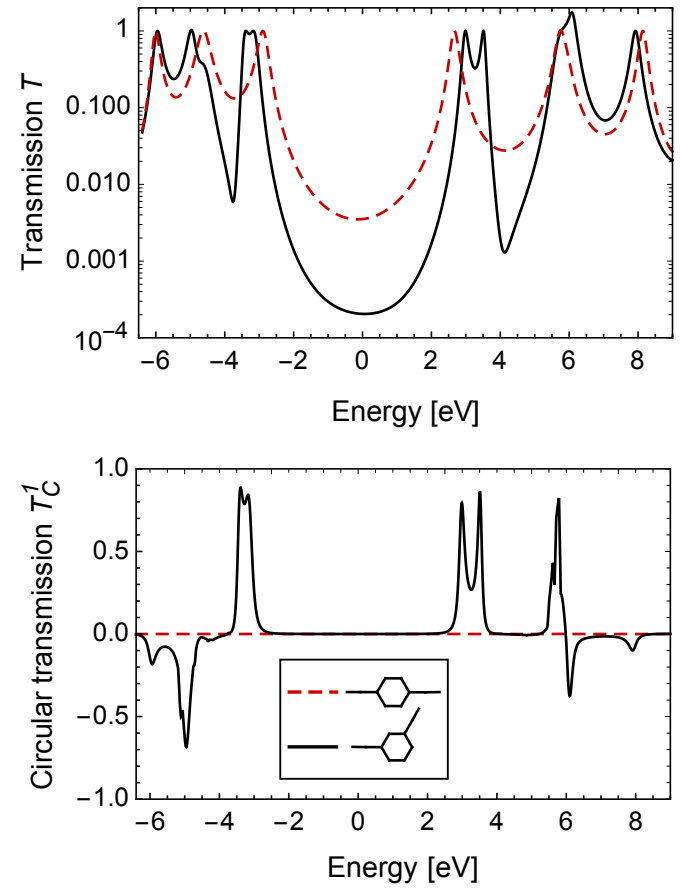

Figure 3. Calculated transmission $T$ (top) and circular transmission $T_{C}^{1}$ (bottom, calculated by equation $(60)$ in the benzene molecule with leads in the meta (black curve) and the para (red dashed curve) configuration. The transmission shows in both cases band gaps, resonances as well as antiresonances. Note that the middle between the HOMO and LUMO resonance energies has been chosen as the origin of the energy scale. Strong circular currents can be observed for certain electron energies in the meta configuration, while in the para configuration no current vortices exist.

\section{RESULTS \& DISCUSSION}

\section{Benzene}

Let us begin our discussion with the simplest aromatic carbon molecule, namely benzene. The transmission calculated by means of the DFT-NEGF method is shown in Figure 3 (top) for the para as well as for the meta configuration of the leads. In both configurations, transmission band gaps, resonances as well as anti-resonances can be observed. Instead of the global transport properties of the molecular junction, which have been addressed previously [4, we rather investigate here on which pathways the current flows through the system. Typical transmission pathways, which can be observed by varying the electron energy, are shown in Figure 4 by means of arrows of different size and color shading. Note that these local transmissions are normalized with respect to the maximum value at each electron energy (or microwave frequency) and hence, do not reflect the absolute transmission through the molecule. Moreover, transmissions less than $10 \%$ of the maximal value are not plotted. 
Starting with the para configuration, we observe that the normalized transmission pathways are independent of the electron energy and split up into two paths that carry equal amounts of current. These properties can be understood easily by the mirror symmetry of the molecular junction with respect to the horizontal axis through the center of the carbon hexagon, which implicates identical currents in both paths and hence, circular currents are not possible.

In the meta configuration, we observe that within the transmission band gap $(E \sim 0 \mathrm{eV})$, the current is flowing on the shortest path between source and drain. Such asymmetric transmission pathways are possible as the molecular junction is no longer mirror symmetric with respect to the horizontal axis. If we change the electron energy to the transmission resonances, we observe a circular current that is rotating around the carbon ring. Passing through the antiresonance (at $E \sim$ $4 \mathrm{eV}$ ), the direction of rotation changes. The full dependence of the transmission pathways on the electron energy (or microwave frequency) is shown in the three movies Benzene-DFT.mov for the DFT-NEGF calculations, Benzene-Experiment.mov for the microwave emulations experiment, and Benzene-TB .mov for calculations within a simple tight-binding model. These movies can be accessed by clicking on the file names.

The circular transmission pathways in the carbon hexagon $\alpha$ are quantified by $[20,42$

$$
T_{C}^{\alpha}(E)=\frac{1}{L_{\alpha}} \sum_{\substack{i, j \in \alpha \\ i<j}} T_{i j}(E) \operatorname{sign}\left(\left(\boldsymbol{r}_{i}-\boldsymbol{r}_{\alpha}\right) \times\left(\boldsymbol{r}_{j}-\boldsymbol{r}_{\alpha}\right)\right)
$$

where $T_{i j}(E)$ is the local transmission between two carbon atoms located at the positions $\boldsymbol{r}_{i}$ and $\boldsymbol{r}_{j}$. The sum is over all atoms which are part of the hexagon $\alpha$ with center $\boldsymbol{r}_{\alpha}$ and length $L_{\alpha}$. The circular transmission as a function of the electron energy is shown in Figure 3 (bottom). The circular transmission, calculated in terms of the normalized transmissions, is indicated in Figure 4 as the color shading of the carbon hexagons. Both figures confirm the existence of circular currents in meta-benzene for certain energies ranges, whereas no current vortices exist in para-benzene.

The circular transmissions give rise to a magnetic field passing through the carbon rings. In order to estimate its strength, we integrate $T_{C}^{1}(E)$ around the double resonance at $3 \mathrm{eV}$ within a bias window of $2 \mathrm{eV}$ (to be precise, in the energy range from $2 \mathrm{eV}$ to $4 \mathrm{eV}$ ) and obtain an absolute circular current of approximately $I_{C} \sim 30 \mu \mathrm{A}$, which in turn causes in the center of the carbon hexagon a magnetic field ${ }^{1}$ ff $B \sim 150 \mathrm{mT}$. Note that the circular transmission in equation (6) is defined in such a way

\footnotetext{
${ }^{1}$ The magnetic field on an axis, which goes perpendicular through
}

that it is the only source of the magnetic field that is passing through the ring. In other words, the remaining transverse current does not generate any mangetic flux, see Ref. 20, 42 for details. Transmission pathways like those at $E=0 \mathrm{eV}$ and $E=-5 \mathrm{eV}$ are also considered as current vortices, because they have a finite circular transmission and give rise to a magnetic field. However, this magnetic field will be weaker and more inhomogenous than in the cases where the current is circulating uni-directionally around the complete carbon ring as at $E=3.5 \mathrm{eV}$.

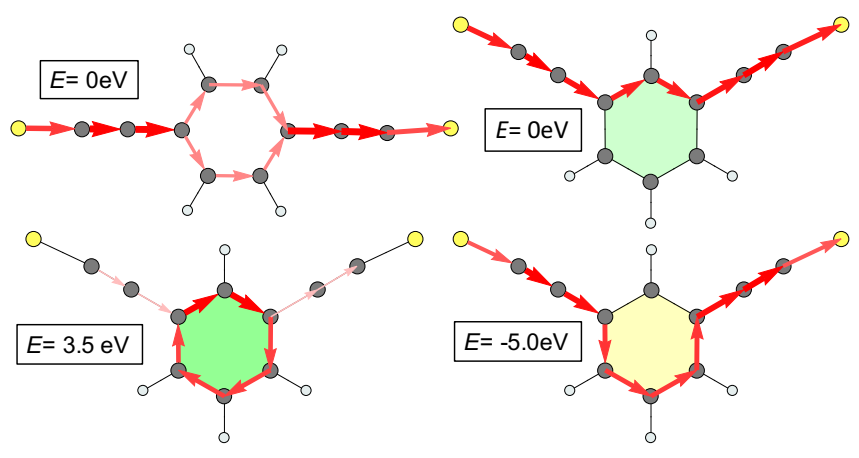

Figure 4. Normalized transmission pathways (arrows) in the benzene molecular junction, calculated by the DFT-NEGF method. The carbon, hydrogen and sulfur atoms are represented by dark gray, light gray and yellow disks, respectively. The gold clusters (see Figure 1) are not shown. The size and color shading of the arrows indicate the magnitude of the local transmissions. The color shading of the hexagons indicates the normalized circular transmissions $T_{C}^{1}$ (see equation (6)); greenish colors for clockwise circulation, yellowish colors for the opposite direction. For the above shown transmission pathways the full dependence on the electron energy is shown in the movie Benzene-DFT.mov

Now, let us turn to the data from the microwave emulation experiment. Figure 5 shows the transmission $T$ and circular transmission $T_{C}^{1}$ as a function of the microwave frequency as well as the (normalized) transmission pathways for various microwave frequencies. The transmission shows several resonances and anti-resonances, similar to the DFT-NEGF calculations. However, their number differs because the microwave experiment emulates only the non-interacting $\pi$ electron system, while in the DFT-NEGF calculations all electrons are taken into account. The emulation experiment shows a considerable reduction of the transmission (in the range from -10 to

the center of a carbon hexagon, is given by

$$
B(z)=\frac{3 \sqrt{3} \mu_{0} I_{C}}{4 \pi a} \frac{1}{\left[(z / a)^{2}+3 / 4\right] \sqrt{(z / a)^{2}+1}},
$$

where $a \sim 0.139 \mathrm{~nm}$ is the distance of the carbon atoms and $I_{C}$ is the current circulating in the hexagon. 
$30 \mathrm{MHz}$ ) in the meta configuration, which resembles the band gap observed in the DFT-NEGF calculations. In the para configuration, the transmission is rather constant while the DFT-NEGF calculations show a (though less pronounced) band gap. This discrepancy can be explained again by the fact that only a part of the electronic structure is emulated by the experiment, because the simple tight-binding model discussed below shows similar trends as the emulation experiment, see Figure 7. Moreover, the overall transmission is lower and its features are much less pronounced than in the calculations, which can be explained by the overall absorption of the microwaves [56, 57]. Note that the transmission is measured between the antenna at the left end, see Figure 2 , and the antenna on top of the next-to-last site at the right end. If the antenna is placed on top of the last site at the right end, the transmission that is passing through the absorber is measured. It follows the same trends as the transmission to the next-to last site but is damped strongly (approximately one order of magnitude).

The transmission pathways in the meta-benzene analogue show strong circular currents at certain frequencies and agree qualitatively with the patterns obtained in the DFT-NEGF study, although a sign reversal of the circular transmission is not found. In para-benzene, we find transmission pathways that are similar to the theoretical predictions but surprisingly, we also observe asymmetric patterns as well as current vortices. Note that in both cases the local transmissions are not conserved due to the absorption of the microwaves. In particular, the transmissions towards the drain at the right end decay strongly, because of the absorber on top of the last resonator, which damps the current that is passing to the measuring antenna (on top of this resonator).

In order to better understand the observed effects, let us consider a simple nearest-neighbor tight-binding model for the benzene molecule. The Hamiltonian, its eigenenergies and eigenstates are shown in Figure 6. By means of these eigenstates, we can calculate the current that is flowing in the isolated molecule (i.e. in absence of the leads) at the corresponding eigenenergies. The non-degenerate states at $E= \pm 2$ do not cause any current flow, because they are entirely real. The degenerate states at $E= \pm 1$ are complex valued and generate non-zero ring currents with opposite direction of rotation. The same observation holds for the angular momentum ${ }^{2}$

2 The expected value of the angular momentum of a tight-binding state $\psi$ is determined by

$$
\langle\psi|L| \psi\rangle \sim \sum_{i<j} \operatorname{Im}\left(H_{i j}^{*} \psi_{i}^{*} \psi_{j}\right)\left(\left(\boldsymbol{r}_{i}-\boldsymbol{r}_{0}\right) \times\left(\boldsymbol{r}_{j}-\boldsymbol{r}_{0}\right)\right),
$$

where $\boldsymbol{r}_{i}$ are the positions of the sites (or atoms) with respect to the center $\boldsymbol{r}_{0}$. The angular momentum is proportional to the circular current in equation $\sqrt{6}$ because $G_{i j}^{n} \sim \psi_{i}^{*} \psi_{j}$.
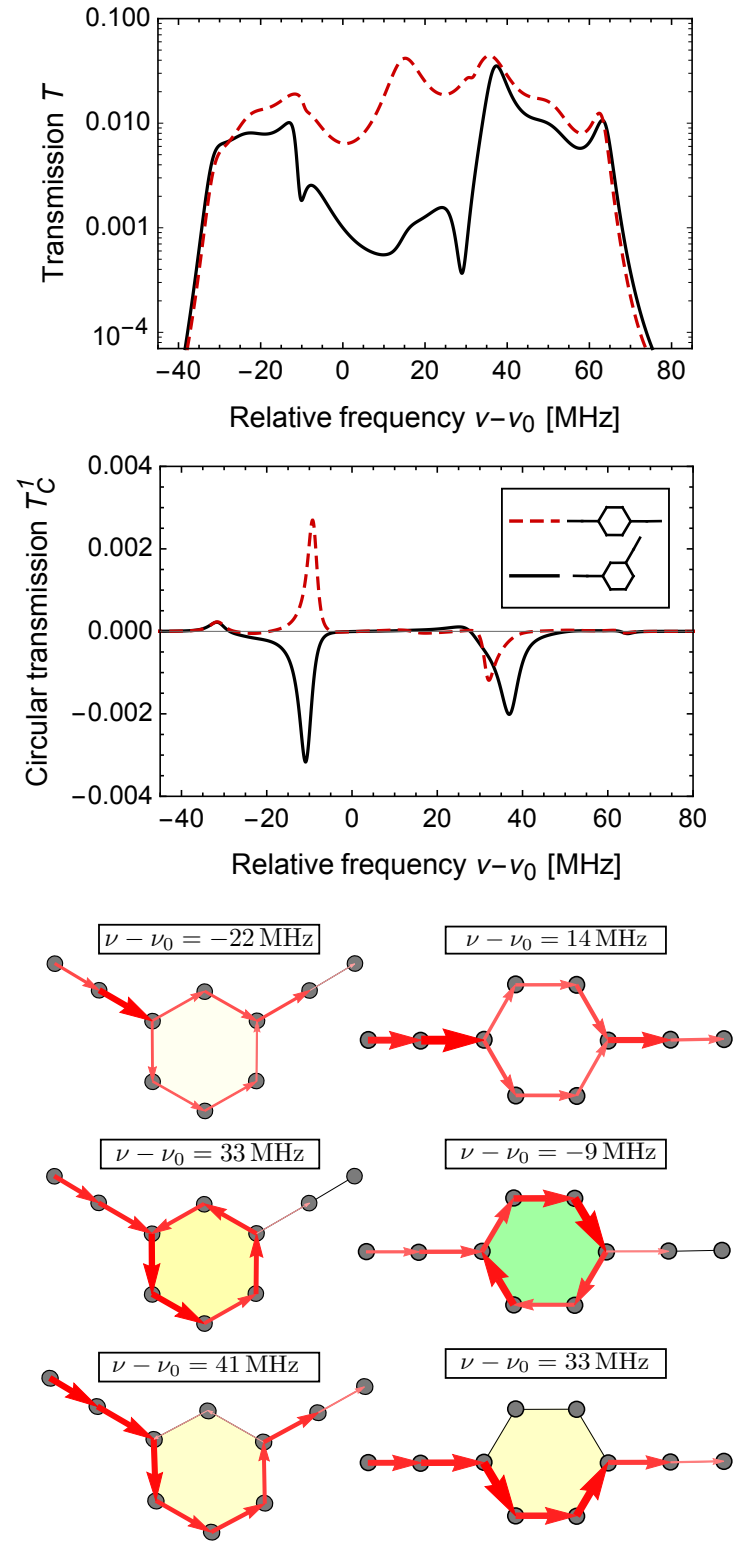

Figure 5. Current flow in the microwave emulation experiment. Top: Transmission and circular transmission as a function of the microwave frequency. Bottom: Normalized transmission pathways at different microwave frequencies. The microwave experiment clearly confirms the existence of circular currents. The transmission pathways as a function of the microwave frequency can be found in the movie Benzene-Experiment.mov

of these states as it is proportional to the circular current in equation (6) [20, 42]. However, note that one could choose the degenerate eigenstates also in such a way that their ring currents and angular momenta are zero.

The coupling of the molecule to the leads is modelled within the wide-band approximation with the selfenergies

$$
\Sigma_{i j}^{S / D}=-\mathrm{i} \eta \delta_{i, j} \delta_{i, s / d}
$$




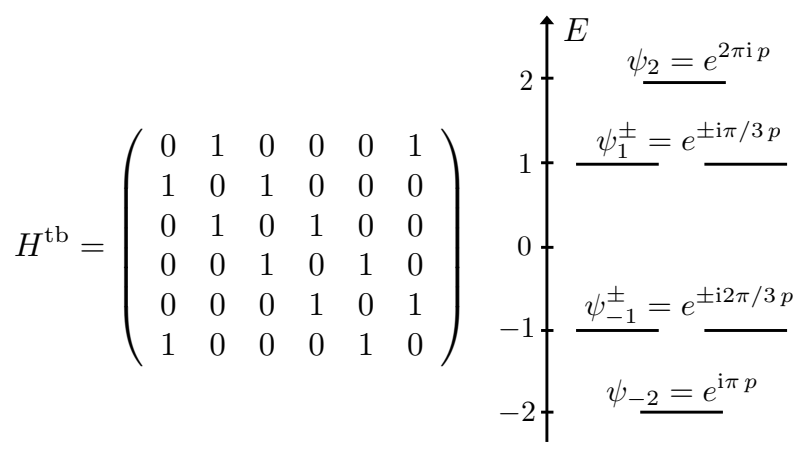

Figure 6. Left: nearest-neighbor tight-binding model of benzene. Right: eigenenergies and eigenstates. The variable $p \in(0,1,2,3,4,5)$ numerates cyclically the six carbon atoms and generates the six componentes of the eigenstates.
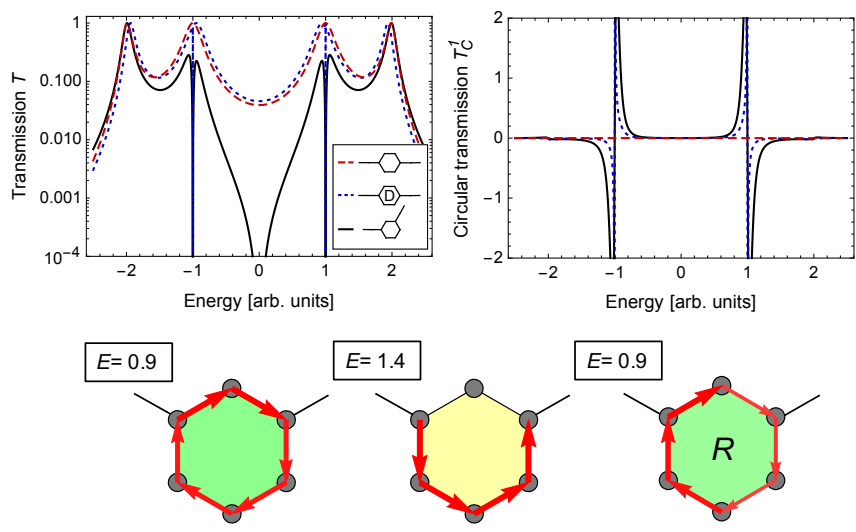

$E=0.9$
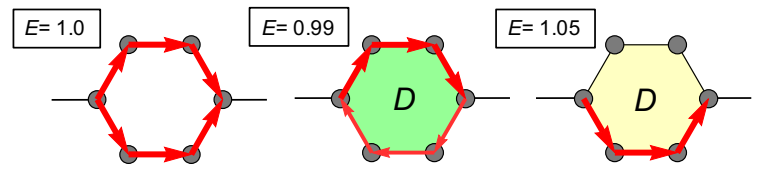

Figure 7. Current flow in the benzene molecule modelled by a simple tight-binding Hamiltonian (Figure 6) and wide-band leads (equation (7)). The transmission and circular transmission (top) show similar features as the microwave emulation experiment. The transmission pathways (bottom) show vortices that are obtained also in the DFT-NEGF calculations and the experiment. The pathway with the label $R$ has been obtained by taking into account only the two eigenstates close to $E=1$. For the pathways with the label $D$, we have introduced random fluctuations (5\%) in the coupling energies, which induces circular currents. The full dependence of the transmission pathways as a function of the electron energy can be found in the movie Benzene-TB.mov.

where $s=1$ is the position of the source, while $d=4$ and $d=3$ give the position of the drain in the para and meta configuration, respectively. The parameter $\eta=0.2$ controls the coupling strength of the leads and $\delta_{i j}$ is the Kronecker delta function. This non-Hermitian perturbation of the system lifts on the one hand the degeneracies of the eigenstates, but on the other hand all of these eigenstates have zero angular momentum. How- ever, the transmission pathways calculated by means of equation (3) agree qualitatively with the DFT-NEGF calculations and the microwave experiment and confirm the existence of circular currents, see Figure 7. An analytic study of the benzene molecular junction that focusses on quantum interference can be found in Ref. 66].

To understand the existence of these vortices, we expand equation (3) in terms of the eigenstates $\psi_{\alpha}$ of the open system $H+\Sigma_{S}+\Sigma_{D}$,

$$
T_{i j}(E)=\sum_{\alpha, \beta} \operatorname{Im}\left[H_{i j}^{*} \frac{\psi_{\alpha, s} \psi_{\alpha, i} \psi_{\beta, j}^{*} \psi_{\beta, s}^{*}}{\left(E-\varepsilon_{\alpha}\right)\left(E-\varepsilon_{\beta}^{*}\right)}\right]
$$

where $s=1$ is the position of the source. Let us start with the meta configuration. Taking into account in the sum in equation (8) only the two eigenstates close to $E= \pm 1$, which are degenerate in absence of the leads, we observe qualitatively the same circular transmission pathways, see in Figure 7 the benzene molecule marked with $R$. Taking into account in equation (8) only the diagonal terms (i.e. $\alpha=\beta$ ), or considering only one of the two eigenstates, gives qualitatively different (and hence incorrect) transmission pathways without any ring currents. In para benzene, where ring currents are not possible due to the symmetry of the molecular junction (see above), one of the degenerate eigenstates at $E= \pm 1$ (i.e. the state $\left.\psi_{ \pm 1}^{\prime}=\psi_{ \pm 1}^{+}-\psi_{ \pm 1}^{-}\right)$is unchanged by the leads, because it has zero value on the carbon atoms where the leads are attached. This eigenstate does not couple to the leads and does not contribute to the current flow. The current around $E= \pm 1$ is determined by a single eigenstate and does not show any vortices. The reader maybe intereded in a discussion of related problems using molecular orbitals in Refs. 34 .

The fact that the microwave experiment shows circular currents also in the para configuration of the benzene molecule can be explained by the imperfections of the experiment. The resonance frequency of the dielectric resonators $( \pm 5 \mathrm{MHz})$ as well as their position $( \pm 0.2 \mathrm{~mm})$ vary slightly due to manufacturing imperfections and limited precision in placing them, respectively. We take into account these imperfections in the tight-binding calculations by changing randomly the nearest-neighbor coupling energies by $5 \%$. This induces circular currents also in para benzene, see the transmission pathways with the label $D$ in Figure 7. In terms of equation (8), the random perturbations couple the (previously uncoupled) eigenstates $\psi_{ \pm 1}^{\prime}$ to the leads and hence, induces current vortices. As the perturbations are small, the coupling is weak and ring currents are observed only in a narrow window around $E= \pm 1$. Taking into account this type of disorder in the meta configuration does not change qualitatively the observed current patterns, because a priori all eigenstates are coupled to the leads. 

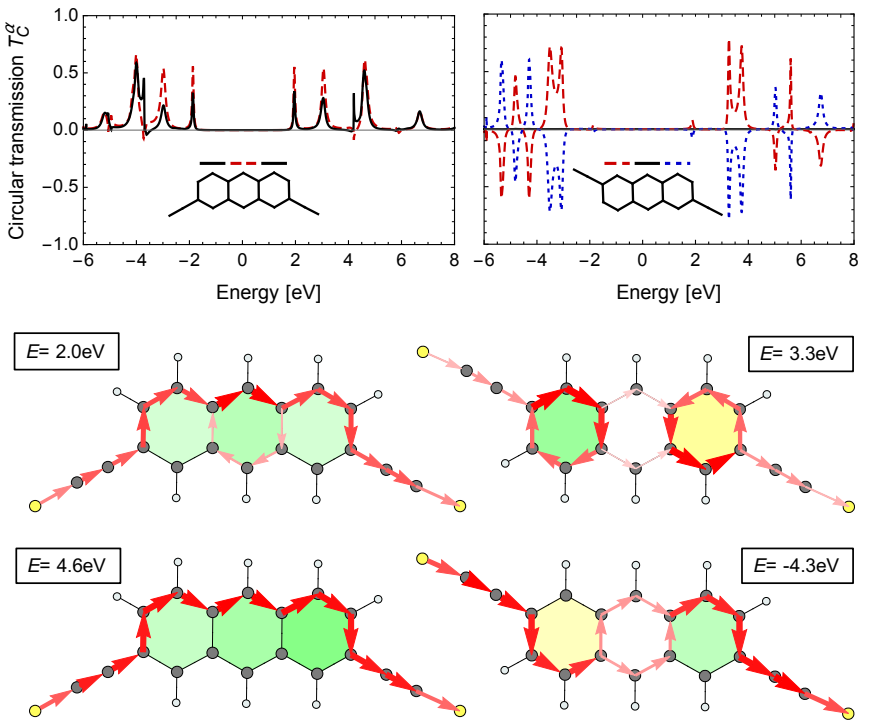

Figure 8. Current flow in the anthracene molecular junction calculated by means of the DFT-NEGF method. The meta configuration is shown in the left column, the para configuration in the right column. Top: Circular transmission in the three $(\alpha \in(1,2,3))$ carbon rings. Bottom: Transmission pathways at various electron energies.

\section{Anthracene \& naphthalene}

Now, let us proceed with longer carbon ring molecules, namely anthracene and naphthalene. The circular transmission and the transmission pathways, calculated by the DFT-NEGF method, are shown in Figure 8 for the meta and para configuration of the anthracene molecular junction. The corresponding results from the microwave emulation experiment are shown in Figure 9. Theory and experiment confirm both the existence of circular currents for the two configurations of the leads.

The circular currents can be understood by a simple tight-binding Hamiltonian, similar to our discussion of the benzene molecule, see Figure 6. In anthracene, we have four two-fold degenerate eigenstates at the energies $E= \pm 1$ and $E= \pm \sqrt{2}$, where strong current vortices can appear, see Figure 10. Let us consider a pair of these degenerate states. When the leads are attached to the molecule, the degeneracy is lifted but only in the para configuration both states couple to the leads. Taking into account in equation (8) only these two states shows that they determine the transmission pathways in the proximity of their eigenenergy. In particular, the correlations between the states, taken into account in equation (8) by the elements with $\alpha \neq \beta$, contribute significantly to the current flow. When the leads are attached in the meta position, one of the previously degenerate states has zeros at the positions of source and drain and hence, does not couple to them. The fact that in meta anthracene circu-
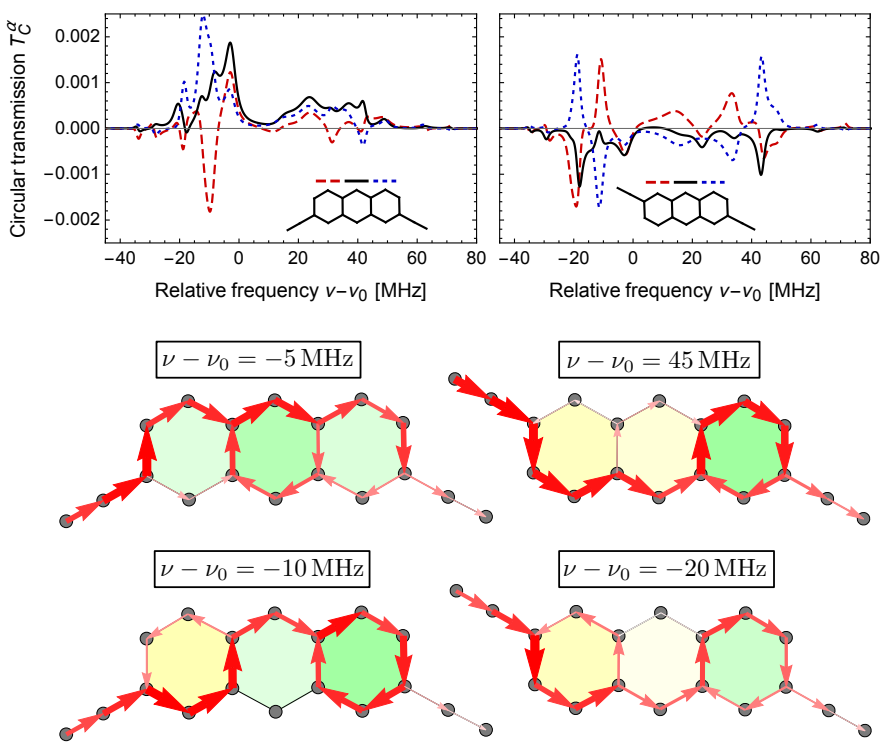

Figure 9. Circular transmission (top) and transmission pathways (bottom) obtained by the microwave emulation experiment of the anthracene molecule. Current vortices are observed in both configurations of the leads.

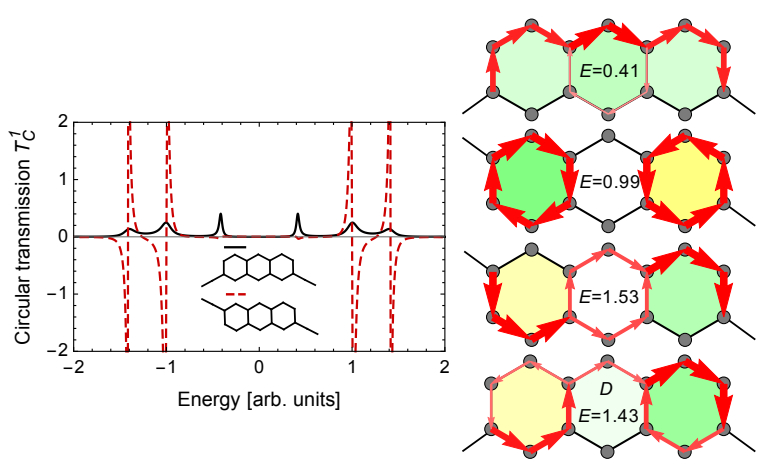

Figure 10. Circular transmission (left) and transmission pathways (right) in the anthracene molecular junction, calculated within a simple nearest-neighbor tight-binding model. The transmission pathways agree qualitatively with the DFTNEGF calculations and the microwave experiment. The asymmetric transmission pathway labeled with $D$ is obtained by changing randomly the coupling energies by $5 \%$.

lar transmission pathways are found, can be explained by the interplay of states that are non-degenerate in the isolated molecule but nearby in energy. This also explains why the circular transmissions is much less pronounced in this configuration of the leads.

In the calculation, the local transmissions and the circular transmission in the three carbon rings show certain symmetries. For example, in the meta configuration the circular transmission in the first and third carbon ring are equal $T_{C}^{1}=T_{C}^{3}$, while in the para configuration they have opposite sign $T_{C}^{1}=-T_{C}^{3}$, and zero value in the central $\operatorname{ring} T_{C}^{2}=0$. These symmetries cannot be observed in 


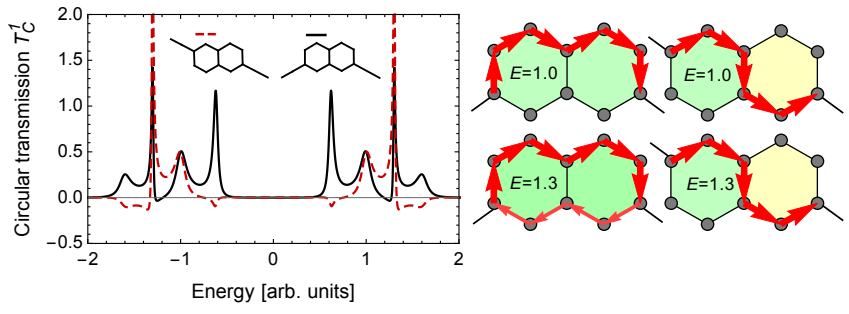

Figure 11. Circular transmission (left) and transmission pathways (right) in the naphthalene molecular junction, calculated within a simple nearest-neighbor tight-binding model. Although all eigenstates of the isolated molecule are nondegenerate, the open system shows circular currents.

the microwave experiment due to the different modelling of source and drain and the imperfections of the experiment (see above). Changing in the tight-binding model randomly the coupling energies by $5 \%$, we obtain asymmetric current patterns very similar to those obtained in the emulation experiment, see for example in Figure 10 the transmission pathway labeled with $D$.

Finally, we address briefly the naphthalene molecule, using a simple tight binding model. All eigenstates of this molecule are non-degenerate and real. Hence, they cannot generate any ring current nor have any angular momentum. However, when leads are coupled to the molecule and transport is studied, circular transmission pathways are observed but their shape differs from the pathways found in benzene and anthracene. In general, they are a consequence of the interplay of all eigenstates of the open system, see equation (8), where the main contributions come from the eigenstates that are nearby the considered energy.

\section{CONCLUSIONS \& OUTLOOK}

We have presented an analysis of the local current flow in benzene and anthracene with some remarks on naphthalene. We have performed state-of-the-art DFT-NEGF calculations of the current flow as well as microwave emulation experiments that model the non-interacting $\pi$ electron system of the molecule. Both show qualitatively similar transmission pathways and confirm the existence of current vortices in certain regions of electron energies, see for example Figures 3 - 5. These emulation experiments are - to the best of our knowledge - the first direct measurement of circular currents in ring structures. The circular currents can be understood in terms of a simple nearest-neighbor tight-binding Hückel model, which also connects more deeply the DFT-NEGF calculations and the emulations experiments, as it approximates the former and describes qualitatively the latter. Using the spectral decomposition of the local transmissions, see equation (8), we have shown that the circular currents can be understood by the interplay of the complex eigenstates with energies close-by the considered electron energy. In particular, the cross terms $(\alpha \neq \beta)$ have a significant effect. Degnerate states, as they appear in benzene and anthracene, generate strong circular currents if both states couple to the leads (like in meta-benzene). One of these states alone cannot induce a current vortex. We have also shown that small imperfections and perturbations can couple previously uncoupled states to the leads and hence, induce current vortices in the system even if symmetry conditions forbid at first their occurrence, see Figure 7 .

As to the significance of the results we can only speculate, but practical use may result from the magnetic field caused by vortices of strong circular transmission. These vortices appear close to transmission maxima and tend to show currents circulating around the complete ring, which imply stronger and more homogeneous magnetic fields within the ring than currents flowing only on one side of the ring. A main challenge to detect this magnetic field is not only to realize experimentally the molecular junctions, but also to shift the Fermi energy close to one of the resonances of the circular transmission [67. We plan to investigate if this can be achieved by designing specific aromatic molecules, for example by adding substituents 68 or by changing the molecular structure 69 . We also expect that current vortices will appear in larger aromatic carbon molecules, like graphene nano-ribbons, which may be easier to implement in the experiment.

\section{CONFLICTS OF INTEREST}

There are no conflicts of interest to declare.

\section{ACKNOWLEDGMENTS}

We gratefully acknowledge funding from CONACYT Proyecto Fronteras 952, UNAM-PAPIIT IA101618, UNAM-PAPIIT AG100819, DFG project HE 5675/5-1 and EU project NEMF21. YPO is gratefull for an UNAM-DGAPA postdoctoral fellowship. We acknowledge use of the MIZTLI super computing facility of DGTIC-UNAM. We thank Reyes Garcia for computer technical support and Thijs Stuyver for his help with the Artaios code.

\section{ELECTRONIC SUPPLEMENTARY MATERIAL}

The transmission pathways in the benzene molecular junction as a function of the electron energy (or microwave frequency) can be found in 
Benzene-DFT.mov for the DFT-NEGF calculations, in Benzene-Experiment.mov for the microwave experiment and in Benzene-TB.mov for the tight-binding calculations.

* stegmann@icf.unam.mx

[1] A. Aviram and M. A. Ratner, Chem. Phys. Lett. 29, 277 (1974)

[2] A. Nitzan and M. Ratner, Science 300, 1384 (2003).

[3] G. Cuniberti and G. R. K. Fagas, eds., Introducing Molecular Electronics (Springer, 2005).

[4] J. C. Cuevas and E. Scheer, Molecular Electronics (World Scientific, 2017).

[5] C. M. Guédon, H. Valkenier, T. Markussen, K. S. Thygesen, J. C. Hummelen, and S. J. van der Molen, Nat. Nanotechnol. 7, 305 (2012).

[6] D. Z. Manrique, C. Huang, M. Baghernejad, X. Zhao, O. A. Al-Owaedi, H. Sadeghi, V. Kaliginedi, W. Hong, M. Gulcur, T. Wandlowski, et al., Nat. Commun. 6, 6389 (2015).

[7] M. Carlotti, A. Kovalchuk, T. Wächter, X. Qiu, M. Zharnikov, and R. C. Chiechi, Nat. Commun. 7, 13904 (2016).

[8] Y. Li, M. Buerkle, G. Li, A. Rostamian, H. Wang, Z. Wang, D. R. Bowler, T. Miyazaki, L. Xiang, Y. Asai, et al., Nat. Mater. 18, 357 (2019).

[9] G. C. Solomon, D. Q. Andrews, T. Hansen, R. H. Goldsmith, M. R. Wasielewski, R. P. V. Duyne, and M. A. Ratner, J. Chem. Phys. 129, 054701 (2008).

[10] T. Markussen, R. Stadler, and K. S. Thygesen, Nano Lett. 10, 4260 (2010).

[11] M. Berritta, D. Z. Manrique, and C. J. Lambert, Nanoscale 7, 1096 (2015).

[12] S. Sangtarash, H. Sadeghi, and C. J. Lambert, Nanoscale 8, 13199 (2016).

[13] X. F. Yang, Y. W. Kuang, Y. S. Liu, D. B. Zhang, Z. G. Shao, H. L. Yu, X. K. Hong, J. F. Feng, X. S. Chen, and X. F. Wang, Nanoscale 8, 15712 (2016).

[14] D. Nozaki and C. Toher, J. Phys. Chem. C 121, 11739 (2017).

[15] Y. Xue and M. A. Ratner, Phys. Rev. B 70, 081404(R) (2004).

[16] M. Ernzerhof, H. Bahmann, F. Goyer, M. Zhuang, and P. Rocheleau, J. Chem. Theory Comput. 2, 1291 (2006).

[17] N. Sai, N. Bushong, R. Hatcher, and M. D. Ventra, Phys. Rev. B 75, 115410 (2007).

[18] G. C. Solomon, C. Herrmann, T. Hansen, V. Mujica, and M. A. Ratner, Nat. Chem. 2, 223 (2010).

[19] C. Herrmann, G. C. Solomon, and M. A. Ratner, J. Phys. Chem. C 114, 20813 (2010).

[20] D. Rai, O. Hod, and A. Nitzan, J. Phys. Chem. C 114, 20583 (2010).

[21] D. Rai, O. Hod, and A. Nitzan, Phys. Rev. B 85, 155440 (2012).

[22] S. K. Maiti, Eur. Phys. J. B 86, 296 (2013).

[23] D. A. Bahamon, Z. Qi, H. S. Park, V. M. Pereira, and D. K. Campbell, Nanoscale 7, 15300 (2015).

[24] F. Al-Dirini, M. A. Mohammed, M. S. Hossain, F. M. Hossain, A. Nirmalathas, and E. Skafidas, Nanoscale 8, 10066 (2016).
[25] P. Lazzeretti, Phys. Chem. Chem. Phys. 18, 11765 (2016).

[26] G. Monaco and R. Zanasi, Phys. Chem. Chem. Phys. 18, 11800 (2016).

[27] D. Sundholm, R. J. F. Berger, and H. Fliegl, Phys. Chem. Chem. Phys. 18, 15934 (2016).

[28] M. Dimitrova, H. Fliegl, and D. Sundholm, Phys. Chem. Chem. Phys. 19, 20213 (2017).

[29] D. Nozaki and W. G. Schmidt, J. Comp. Chem. 38, 1685 (2017).

[30] H. Li, M. H. Garner, T. A. Su, A. Jensen, M. S. Inkpen, M. L. Steigerwald, L. Venkataraman, G. C. Solomon, and C. Nuckolls, J. Am. Chem. Soc. 139, 10212 (2017).

[31] T. Hansen, G. C. Solomon, and T. Hansen, J. Chem. Phys. 146, 092322 (2017).

[32] T. Stuyver, N. Blotwijk, S. Fias, P. Geerlings, and F. De Proft, ChemPhysChem 18, 3012 (2017).

[33] S. Fias and T. Stuyver, J. Chem. Phys. 147, 184102 (2017).

[34] S.-M. Jhan and B.-Y. Jin, J. Chem. Phys. 147, 194106 (2017).

[35] T. Stuyver, S. Fias, P. Geerlings, F. D. Proft, and M. Alonso, J. Phys. Chem. C 122, 19842 (2018).

[36] V. Pohl, L. E. M. Steinkasserer, and J. C. Tremblay, https://arxiv.org/abs/1707.07635

[37] G. Cabra, A. Jensen, and M. Galperin, J. Chem. Phys. 148, 204103 (2018).

[38] M. H. Garner, H. Li, Y. Chen, T. A. Su, Z. Shangguan, D. W. Paley, T. Liu, F. Ng, H. Li, S. Xiao, et al., Nature 558, 415 (2018).

[39] A. Jensen, M. H. Garner, and G. C. Solomon, J. Phys. Chem. C 123, 12042 (2019).

[40] M. H. Garner, A. Jensen, L. O. H. Hyllested, and G. C. Solomon, Chem. Sc. 10, 4598 (2019).

[41] M. Patra and S. K. Maiti, Sci. Rep. 7, 43343 (2017).

[42] U. Dhakal and D. Rai, J. Phys.: Condens. Matter 31, 125302 (2019).

[43] A. Taninaka, S. Yoshida, Y. Sugita, O. Takeuchi, and H. Shigekawa, Nanoscale 11, 5951 (2019).

[44] A. Troisi and M. A. Ratner, Phys. Chem. Chem. Phys. 9, 2421 (2007).

[45] A. Troisi, J. M. Beebe, L. B. Picraux, R. D. van Zee, D. R. Stewart, M. A. Ratner, and J. G. Kushmerick, Proc. Natl. Ac. Sc. 104, 14255 (2007).

[46] J. B. Rix and P. Hedegård, J. Phys. Chem. C 123, 3817 (2019).

[47] H.-J. Stöckmann, Quantum Chaos (Cambridge University Press, 1999).

[48] U. Kuhl, H.-J. Stöckmann, and R. Weaver, J. Phys. A: Math. Gen. 38, 10433 (2005).

[49] D. Laurent, O. Legrand, P. Sebbah, C. Vanneste, and F. Mortessagne, Phys. Rev. Lett. 99, 253902 (2007).

[50] J. A. Franco-Villafañe, E. Sadurní, S. Barkhofen, U. Kuhl, F. Mortessagne, and T. H. Seligman, Phys. Rev. Lett. 111, 170405 (2013).

[51] C. Poli, M. Bellec, U. Kuhl, F. Mortessagne, and H. Schomerus, Nat. Commun. 6 (2015).

[52] U. Kuhl, S. Barkhofen, T. Tudorovskiy, H.-J. Stöckmann, T. Hossain, L. de Forges de Parny, and F. Mortessagne, Phys. Rev. B 82, 094308 (2010).

[53] M. Bellec, U. Kuhl, G. Montambaux, and F. Mortessagne, Phys. Rev. Lett. 110, 033902 (2013).

[54] M. Bellec, U. Kuhl, G. Montambaux, and F. Mortessagne, Phys. Rev. B 88, 115437 (2013). 
[55] J. Böhm, M. Bellec, F. Mortessagne, U. Kuhl, S. Barkhofen, S. Gehler, H.-J. Stöckmann, I. Foulger, S. Gnutzmann, and G. Tanner, Phys. Rev. Lett. 114, 110501 (2015).

[56] T. Stegmann, J. A. Franco-Villafañe, U. Kuhl, F. Mortessagne, and T. H. Seligman, Phys. Rev. B 95, 035413 (2017).

[57] T. Stegmann, J. A. Franco-Villafañe, Y. P. Ortiz, U. Kuhl, F. Mortessagne, and T. H. Seligman, Phys. Lett. A 381, 24 (2017).

[58] M. J. Frisch et al., Gaussian 09 (revision a.02) (2009).

[59] M. Deffner, L. Groß, T. Steenbock, B. A. Voigt, G. C. Solomon, and C. Herrmann, Artaios - a code for postprocessing quantum chemical electronic structure calculations, available from https: //www. chemie. uni-hamburg. de/institute/ac/ arbeitsgruppen/herrmann/software/artaios. html (2008-2019).

[60] C. J. O. Verzijl, J. S. Seldenthuis, and J. M. Thijssen, J. Chem. Phys. 138, 094102 (2013).
[61] S. Datta, Electronic Transport in Mesoscopic Systems (Cambridge University Press, 1997).

[62] A. Pecchia and A. D. Carlo, Rep. Prog. Phys. 67, 1497 (2004).

[63] C. Herrmann, G. C. Solomon, J. E. Subotnik, V. Mujica, and M. A. Ratner, J. Chem. Phys. 132, 024103 (2010).

[64] R. M. Ribeiro, V. M. Pereira, N. M. R. Peres, P. R. Briddon, and A. H. Castro Neto, New J. Phys. 11, 115002 (2009).

[65] P. Seba, U. Kuhl, M. Barth, and H.-J. Stöckmann, J. Phys. A: Math. Gen. 32, 8225 (1999).

[66] T. Hansen, G. C. Solomon, D. Q. Andrews, and M. A. Ratner, J. Chem. Phys. 131, 194704 (2009).

[67] X. Liu, S. Sangtarash, D. Reber, D. Zhang, H. Sadeghi, J. Shi, Z.-Y. Xiao, W. Hong, C. J. Lambert, and S.-X. Liu, Angew. Chem. Int. Ed. 56, 173 (2016).

[68] C. Herrmann, G. C. Solomon, and M. A. Ratner, J. Am. Chem. Soc. 132, 3682 (2010).

[69] H. Schlicke and C. Herrmann, ChemPhysChem 15, 4011 (2014). 